\title{
B Baryon Production and Decays and B Hadron Lifetimes
}

\section{S. Donati*†}

University and INFN Pisa, Largo B. Pontecorvo, 3 - 56127 Pisa, Italy

E-mail: simone.donatiepi.infn.it

In this paper we review the most recent results concerning B Baryons at CDF and D0, including the observation and the study of the properties of the $\Omega_{b}^{-}, \Xi_{b}^{-}$and $\Sigma_{b}^{ \pm(*)}$, the observation of new $\Lambda_{b}^{0}$ decay modes, and a new measurement of the lifetime of the $b$ hadrons in decays with a $J / \psi$. The $\Omega_{b}^{-}$baryon is observed through the decay chain $\Omega_{b}^{-} \rightarrow J / \psi \Omega^{-}$, where $J / \psi \rightarrow \mu^{+} \mu^{-}$, $\Omega^{-} \rightarrow \Lambda K^{-}$, and $\Lambda \rightarrow p K^{-}$, using $4.2 \mathrm{fb}^{-1}$ of data. The $\Omega_{b}^{-}$mass is measured to be $6054.4 \pm 6.8$ (stat.) \pm 0.9 (syst.) $\mathrm{MeV} / \mathrm{c}^{2}$, and the lifetime $1.13_{-0.40}^{+0.53}$ (stat.) \pm 0.02 (syst.) ps.

For the $\Xi_{b}^{-}$the mass is measured $5790.9 \pm 2.6$ (stat.) \pm 0.8 (syst.) $\mathrm{MeV} / \mathrm{c}^{2}$ and the lifetime $1.56_{-0.25}^{+0.27}$ (stat.) \pm 0.02 (syst.) ps.

A new accurate measurement of the properties of the resonances $\Sigma_{b}^{+}, \Sigma_{b}^{-}, \Sigma_{b}^{*+}$, and $\Sigma_{b}^{*-}$ has been performed in $6 \mathrm{fb}^{-1}$ of data, and the masses have been determined, $m\left(\Sigma_{b}^{+}\right)=5811.2_{-0.8}^{+0.9}$ (stat.) \pm 1.7 (syst. $), \quad m\left(\Sigma_{b}^{-}\right)=5815.5_{-0.5}^{+0.6}$ (stat.) \pm 1.7 (syst.), $m\left(\Sigma_{b}^{*+}\right)=$ $5832.0 \pm 0.7$ (stat.) \pm 1.8 (syst.), and $m\left(\Sigma_{b}^{*-}\right)=5835.0 \pm 0.6$ (stat.) \pm 1.8 (syst.).

The $\Lambda_{b}^{0} \rightarrow \Lambda_{c}^{+} \pi^{-} \pi^{+} \pi^{-}$decay mode has been observed in $2.4 \mathrm{fb}^{-1}$ of data, with the resonant decay modes $\Lambda_{b}^{0} \rightarrow \Lambda_{c}(2595)^{+} \pi^{-} \rightarrow \Lambda_{c}^{+} \pi^{-} \pi^{+} \pi^{-}, \Lambda_{b}^{0} \rightarrow \Lambda_{c}(2625)^{+} \pi^{-} \rightarrow \Lambda_{c}^{+} \pi^{-} \pi^{+} \pi^{-}, \Lambda_{b}^{0} \rightarrow$ $\Sigma_{c}(2455)^{++} \pi^{-} \pi^{-} \rightarrow \Lambda_{c}^{+} \pi^{-} \pi^{+} \pi^{-}$, and $\Lambda_{b}^{0} \rightarrow \Sigma_{c}(2455)^{0} \pi^{+} \pi^{-} \rightarrow \Lambda_{c}^{+} \pi^{-} \pi^{+} \pi^{-}$.

$\mathrm{CDF}$ has performed new measurements of the $b$ hadron lifetimes in decays with a $J / \psi$. The measured lifetimes are the currently most precise determination of the $B^{+}$, $B^{0}$ and $\Lambda_{b}^{0}$ lifetimes. The measured values are $c \tau\left(B^{+}\right)=491.4 \pm 2.6$ (stat.) \pm 2.6 (syst.) $\mu \mathrm{m}$, $c \tau\left(B^{)}\right)=451.7 \pm 3.0$ (stat.) \pm 2.5 (syst.) $\mu \mathrm{m}$, and $c \tau\left(\Lambda_{b}^{0}\right)=460.8 \pm 13.4$ (stat.) \pm 4.1 (syst.) $\mu \mathrm{m}$.

FPCP $2010-8^{\text {th }}$ Flavour Phyics and CP Violation 2010

May 25 - 29, 2010

Torino, Italy

\footnotetext{
* Speaker.

${ }^{\dagger}$ for the CDF and D0 Collaborations
} 


\section{Introduction}

B hadrons are abundantly produced at the Tevatron Collider, where the measured $b$ production cross section is $\sigma\left(B^{+}\right)=2.78 \pm 0.24 \mu \mathrm{b}$ for $p_{T}\left(B^{+}\right) \geq 6 \mathrm{GeV} / \mathrm{c}$ and $|y| \leq 1$ ([1]), and the available energy allows the production of the heavier $\Lambda_{b}, \Sigma_{b}, \Xi_{b}$ and $\Omega_{b}$ hadrons. The challenge is extracting signals from background which are orders of magnitude higher at production. This is achieved with dedicated detectors and triggers.

The CDF II tracker is made of three silicon detectors ([3]) and a drift chamber ([4]) located within a solenoidal magnetic field. Particle identification is performed with the measurement of the specific ionisation in the drift chamber and of the time of flight in a specific detector ([5]). Segmented electromagnetic and hadronic calorimeters surround the tracking system ([6]). The muon detectors ([7]) are located outside the central hadron calorimeter. CDF II uses a three-level trigger system. The heart of the L1 trigger is the eXtremely Fast Tracker ([8]), the trigger track processor that identifies charged tracks in the drift chamber. The L1 tracks are extrapolated to the calorimeter and to the muon chambers to generate electron and muon trigger candidates. The Online Silicon Vertex Tracker ([9]) is part of the L2 trigger. It receives the L1 tracks and the digitised pulse heights on the axial layers of the silicon vertex detector. It links the L1 tracks to the silicon hits and reconstructs tracks with offline-like quality, used to select online the secondary vertices characteristic of the $b$ events in specific triggers. L3 trigger uses a CPU farm which allows to perform an almost offline-quality reconstruction.

The D0 detector [10] uses an excellent central tracking system which consists of a silicon microstrip tracker and a central fiber tracker surrounded by a solenoidal magnet and provides signals to the Level 2 and Level 3 trigger systems to select events with displaced vertices from $b$-quark decay. The D0 calorimeter system consists of three sampling calorimeters (primarily uranium/liquidargon) and an intercryostat detector.The muon system uses proportional drift tubes, mini drift tubes, and toroidal magnets and provides a coverage to $|\eta| \approx 2.0$.

\section{Observation and properties of the $\Omega_{b}^{-}$and $\Xi_{b}^{-}$baryons}

The $\Omega_{b}^{-}$observation is made at $\mathrm{D} 0[12]$ and $\mathrm{CDF}[11]$ through the decay chain $\Omega_{b}^{-} \rightarrow J / \psi \Omega^{-}$, where $J / \psi \rightarrow \mu^{+} \mu^{-}, \Omega^{-} \rightarrow \Lambda K^{-}$, and $\Lambda \rightarrow p \pi^{-}$. The $\Xi_{b}^{-}$is reconstructed through the similar decay mode $\Xi_{b}^{-} \rightarrow J / \psi \Xi^{-}$, where $J / \psi \rightarrow \mu^{+} \mu^{-}, \Xi^{-} \rightarrow \Lambda \pi^{-}$, and $\Lambda^{-} \rightarrow p \pi^{-}$as a crosscheck. The CDF analysis selects well-measured $J / \psi \rightarrow \mu^{+} \mu^{-}$candidates, where the two-muon invariant mass is required within $80 \mathrm{MeV} / \mathrm{c}^{2}$ of the world-average $J / \psi$ mass. $\Lambda$ candidates use all opposite charge track pairs with $p_{T}>0.4 \mathrm{GeV} / \mathrm{c}$ found in the chamber. The proton (pion) mass is assigned to the track with the higher (lower) momentum, which is correct for the $\Lambda$ candidates used in this analysis for the kinematics of the $\Lambda$ decay and the lower limit in the transverse momentum acceptance of the tracking system. The additional tracks are assigned the pion or kaon mass, and $\Lambda \pi^{-}$and $\Lambda K^{-}$ combinations are identified that are consistent with the decay process $\Xi^{-} \rightarrow \Lambda \pi^{-}$or $\Omega^{-} \rightarrow \Lambda K^{-}$. The charged hyperon candidates have an additional fit performed with the three tracks that simultaneously constrains the $\Lambda$ and $\Xi^{-}$or $\Omega^{-}$masses of the appropriate track combinations and provides the best possible estimate of the hyperon momentum and decay position. A significant background reduction is achieved by requiring the charged hyperon candidates have track measurements in at 
least one layer of the silicon detector. The shorter lifetime of the $\Omega^{-}$makes the silicon selection not efficient in comperate to the $\Xi^{-}$. For this reason silicon detector information on the hyperon track is used when it is available, but it is not imposed as a requirement for the $\Omega^{-}$selection. The hyperon candidates are combined with the $J / \psi$ candidates by fitting the five-track state with constraints approprate for each decay topology and intermediate hadron state. The $\mu^{+} \mu^{-}$mass is constrained to the nominal $J / \psi$ mass, and the hyperon candidate is constrained to originate from the $J / \psi$ decay vertex. The fits that include the charged hyperon constrain the $\Lambda$ candidate tracks to the nominal $\Lambda$ mass, and the $\Xi^{-}$and $\Omega^{-}$candidates to the respective nominal masses. $b$-hadron candidates are required to have $p_{T}>6.0 \mathrm{GeV} / \mathrm{c}$ and the hyperon to have $p_{T}>2.0 \mathrm{GeV} / \mathrm{c}$. Figure 1 shows the $\operatorname{CDF} \Xi_{b}^{-}$and $\Omega_{b}^{-}$mass distributions.

CDF measures a $\Xi_{b}^{-}$mass to be $5790.9 \pm 2.6$ (stat.) \pm 0.8 (syst.) $\mathrm{MeV} / \mathrm{c}^{2}$ and the $\Omega_{b}^{-}$to be $6054.4 \pm 6.8$ (stat.) \pm 0.9 (syst.) $\mathrm{MeV} / \mathrm{c}^{2}$. The systematic errors are due to the uncertainty on the mass scale of the baryons measured with the hyperons in the final state, estimated as the mass difference between the $B^{0}$ as measured in the $J / \psi K_{s}^{0}$ and the nominal $B^{0}$ mass and rescaled for the different energy measured by the tracking system in the two decay modes. A systematic error is due to the dependence of the measured on the alternative assumption to have a constant or an event-by-event mass resolution in the fit. A further systematic is due to the uncertainty on the $\Omega^{-}$mass. The $\Xi_{b}^{-}$lifetime is measured to be $1.56_{-0.25}^{+0.27}$ (stat.) \pm 0.02 (syst.) ps and the lifetime of the $\Omega_{b}^{-}$to be $1.13_{-0.40}^{+0.53}$ (stat.) \pm 0.02 (syst.) ps. The systematic errors are due to the treatment of the resolution on the proper decay length in the fit $(2 \mu \mathrm{m})$, to the detector mis-alignment $(1 \mu \mathrm{m})$. The productions of the $\Xi_{b}^{-}$and of the $\Omega_{b}^{-}$relative to the $\Lambda_{b}^{0}$ are found to be $\frac{\sigma\left(\Xi_{b}^{-}\right) B R\left(\Xi_{b}^{-} \rightarrow J / \psi \Xi^{-}\right)}{\sigma\left(\Lambda_{b}^{0}\right) B R\left(\Lambda_{b}^{0} \rightarrow J / \psi \Lambda\right)}$ $=0.167_{-0.025}^{+0.037}$ (stat.) \pm 0.012 (syst.), and $\frac{\sigma\left(\Omega_{b}^{-}\right) B R\left(\Omega_{b}^{-} \rightarrow J / \psi \Omega^{-}\right)}{\sigma\left(\Lambda_{b}^{0}\right) B R\left(\Lambda_{b}^{0} \rightarrow J / \psi \Lambda\right)}=0.045_{-0.012}^{+0.017}$ (stat.) \pm 0.004 (syst.). The acceptance and reconstruction efficiency, determined from Monte Carlo, depends on the $p_{T}$ distribution at production of the $\Xi_{b}^{-}$and $\Omega_{b}^{-}$. The analysis assumes that the $\Xi_{b}^{-}$and $\Omega_{b}^{-}$are produced with the same $p_{T}$ distribution as the $\Lambda_{b}^{0}$. The uncertainty on the efficiencies contains contributions due to $f_{\Lambda_{b}^{0}}$, to the Monte Carlo samples size, to the simulation of the tracking system, to the $\Xi^{-}$and $\Omega^{-}$branching fractions, to the uncertainty on the $\Lambda_{b}^{0}$ yield.

D0 performed the first observation of the $\Xi_{b}^{-}$in $1.3 \mathrm{fb}^{-1}$ of data [13] and measured the mass to be $5774 \pm 11$ (stat.) \pm 15 (syst.) $\mathrm{MeV} / \mathrm{c}^{2}$. D0 observed also a signal of $17.8 \pm 4.9$ events of $\Omega_{b}^{-}$with a significance of $5.4 \sigma$ is the same decay mode as CDF. The measured $\Omega_{b}^{-}$mass is $6.165 \pm 10$ (stat.) \pm 13 (syst.) shows a discrepancy of $\approx 6 \sigma$ from CDF. A new analysis which uses five times more statistics is currently in progress at D0 to understand the orgin of this discrepancy.

\section{Measurement of the resonance properties of the $\Sigma_{b}$ and $\Sigma_{b}^{*}$ baryons}

The $\Sigma_{b}$ and $\Sigma_{b}^{*}$ states have been observed at CDF as resonances in the $\Lambda_{b}^{0} \pi^{ \pm}$mass distributions, where $\Lambda_{b}^{0} \rightarrow \Lambda_{c}^{+} \pi^{-}$and $\Lambda_{c}^{+} \rightarrow p K^{-} \pi^{+}$, using $1.1 \mathrm{fb}^{-1}$ of data [14]. The $\Lambda_{b}^{0} \pi$ states are interpreted as the lowest-lying charged $\Sigma_{b}$ baryons and are labeled $\Sigma_{b}^{(*)}$. CDF has recently performed an update of this analysis using $6 \mathrm{fb}^{-1}$ of data. In reconstructing the decays $\Lambda_{b}^{0} \rightarrow \Lambda_{c}^{+} \pi^{-}$and $\Lambda_{c}^{+} \rightarrow p K^{-} \pi^{+}$, the proton from the $\Lambda_{c}^{+}$decay and the $\pi^{-}$from the $\Lambda_{b}^{0}$ decay must have $p_{T}>0.5 \mathrm{GeV} / \mathrm{c} . \Lambda_{c}^{+}$combinatorial background is also suppressed by requiring $p_{T}(p)>p_{T}\left(\pi^{+}\right)$. The $\Lambda_{c}^{+}$daughter tracks are 3-D constrained to originate from a single point and the $\Lambda_{c}^{+}$candidate is constrained to the 

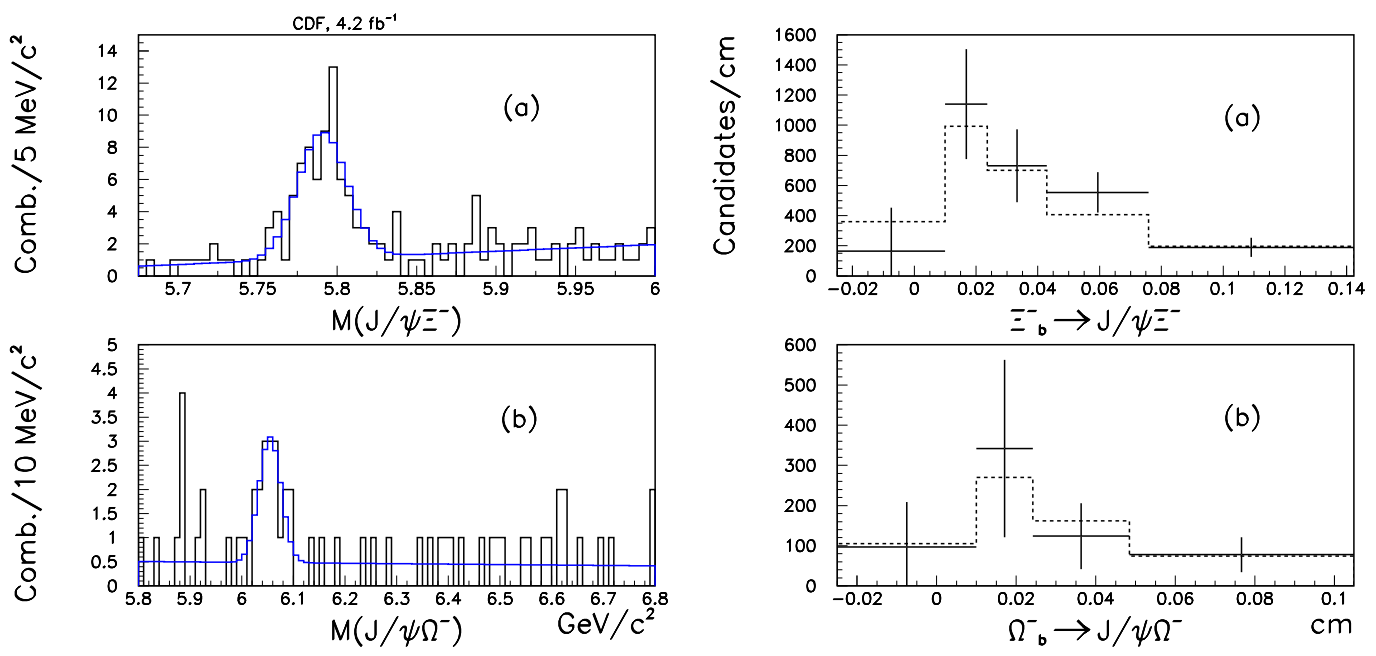

Figure 1: (Left plot) The invariant mass distributions of (a) $J / \psi \Xi^{-}$and (b) $J / \psi \Omega^{-}$combinations for candidates with $c t>100 \mu \mathrm{m}$ reconstructed at CDF. The projections of the unbinned mass fit are indicated by the blue line; (Right plot) The solid histograms represent the number of (a) $\Xi_{b}^{-} \rightarrow J / \psi \Xi^{-}$and (b) $\Omega_{b}^{-} \rightarrow$ $J / \psi \Omega^{-}$candidates found in each $c t$ bin. The dashed histogram is the fit value. (Right plot) $\Lambda_{b}^{0}$ lifetime fit performed on the data.

known $\Lambda_{c}^{+}$mass, and the $\Lambda_{c}^{+}$momentum is extrapolated to intersect the $\pi^{-}$momentum vector to form the $\Lambda_{b}^{0}$ vertex. The $\Lambda_{c}^{+}$and $\Lambda_{b}^{0}$ must have $p_{T}$ above $4.5 \mathrm{GeV} / \mathrm{c}$ and $6.0 \mathrm{GeV} / \mathrm{c}$ respectively. It is also requested $c t\left(\Lambda_{b}^{0}\right)>200 \mu \mathrm{m}$ and the significance $c t\left(\Lambda_{b}^{0}\right) / \sigma_{c t}>12$, and the impact parameter of the $\Lambda_{b}^{0}$ candidate $\left|d_{0}\left(\Lambda_{b}^{0}\right)\right|<80 \mu \mathrm{m}$. The reconstruction of $\Sigma_{b}^{(*)}$ proceeds by combining $\Lambda_{b}^{0}$ candidates in the $\Lambda_{b}^{0}$ signal region with all remaining high quality tracks, with the pion mass hypothesis used when computing the invariant mass of the $\Sigma_{b}^{(*)}$ candidate. Narrow resonances are searched in the mass difference distribution of $Q=m\left(\Lambda_{b}^{0} \pi\right)-m\left(\Lambda_{b}^{0}\right)-m_{\pi}$. Selection cuts are optimised to maximise the sensitivity of the search. Figure 2 reports the $Q$ distributions reconstructed in data. The main source of background is the combination of prompt $\Lambda_{b}^{0}$ baryons,or B mesons reconstructed as $\Lambda_{b}^{0}$ baryons, with extra tracks produced in the hadronization of the $b$ quark. An unbinned maximum likelihood fit determines the mass of the $\Sigma_{b}^{+}=5811.2_{-0.8}^{+0.9}$ (stat.) \pm 1.7 (syst.) $\left(\approx 470\right.$ signal events), the mass of the $\Sigma_{b}^{-}=5815.5_{-0.5}^{+0.6}$ (stat. $) \pm 1.7$ (syst.) $(\approx 330$ signal events), the mass of the $\Sigma_{b}^{*+}=5832.0 \pm 0.7$ (stat.) \pm 1.8 (syst.) $\left(\approx 780\right.$ signal events), and the mass of the $\Sigma_{b}^{*-}=$ $5835.0 \pm 0.6$ (stat.) \pm 1.8 (syst.) $(\approx 520$ signal events). The systematic errors are due to the uncertainty on the fit procedure, on the uncertainties on the momentum scale and on the assumptions made in the fitter, which include the fixed parameters describing the detector resolution and the model describing the background. A more accurate description of this analysis can be found in [15].

\section{Charm baryon spectroscopy}

CDF has performed an analysis of the excited charm baryons $\left(\Lambda_{c}^{+}(2595), \Lambda_{c}^{+}(2625), \Sigma_{c}^{0,++}(2455)\right.$ and $\left.\Sigma_{c}^{0,++}(2520)\right)$ reconstructed in the strong decays to the $\Lambda_{c}^{+}$ground state $\left(\Lambda_{c}^{*+} \rightarrow \Lambda_{c}^{+} \pi^{+} \pi^{-}\right.$and 

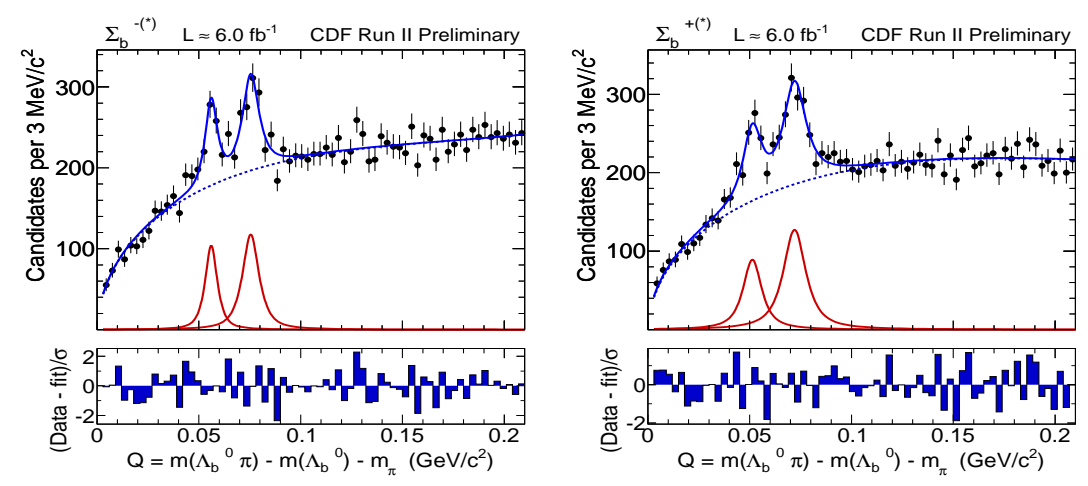

Figure 2: (Left plot) $\Sigma_{b}^{-(*)}$ fit to the $\Lambda_{b}^{0} \pi^{-}$subsample; (Right plot) $\Sigma_{b}^{+(*)}$ fit to the $\Lambda_{b}^{0} \pi^{+}$subsample.

$\left.\Sigma^{0,++} \rightarrow \Lambda_{c}^{+} \pi^{-,+}\right)$. The analysis performs a measurement of the mass differences of these resonances to the $\Lambda_{c}^{+}$mass and of the corresponding decay widths. The $\Lambda_{c}^{+}(2595)$ mass shape is affected by kinematical threshold effects in the resonant subdecay $\Lambda_{c}^{+}(2595) \rightarrow \Sigma_{c}(2455) \pi$ which lead to a mass which is approximately $3 \mathrm{MeV} / \mathrm{c}^{2}$ lower than the previously measured values. This analysis uses the highest number of signal events for all the resonances which leads to the most accurate values of the $\Lambda_{c}^{+}(2595)$ and $\Lambda_{c}^{+}(2625)$ properties[16].

\section{Observation of the resonant structure of the $\Lambda_{b}^{0} \rightarrow \Lambda_{c}^{+} \pi^{-} \pi^{+} \pi^{-}$decay mode}

CDF reconstructed a signal of $848 \pm 93 \Lambda_{b}^{0} \rightarrow \Lambda_{c}^{+} \pi^{-} \pi^{+} \pi^{-}$candidates with $\Lambda_{c}^{+} \rightarrow p K^{-} \pi^{+}$in $2.4 \mathrm{fb}^{-1}$ of data (Figure 3). In the $\Lambda_{b}^{0} \rightarrow \Lambda_{c}^{+} \pi^{-} \pi^{+} \pi^{-}$sample we reconstructed the resonant decay modes: $\Lambda_{b}^{0} \rightarrow \Lambda_{c}(2595)^{+} \pi^{-}$(46.6 \pm 9.7 candidates), $\Lambda_{b}^{0} \rightarrow \Lambda_{c}(2625)^{+} \pi^{-}$(114 \pm 13 candidates), $\Lambda_{b}^{0} \rightarrow \Sigma_{c}(2455)^{++} \pi^{-} \pi^{-}(81 \pm 15$ candidates $)$, and $\Lambda_{b}^{0} \rightarrow \Sigma_{c}(2455)^{0} \pi^{+} \pi^{-}$(41.5 \pm 9.3 candidates) (Figure 3). We measured the relative branching fractions of the resonant $\Lambda_{b}^{0}$ decay modes (Table 1), by using the signal yields estimated by perfoming fits of the mass distributions (Figure 3) and relative efficiency factors estimated with Monte Carlo. The main sources of systematic errors derive from the uncertainties on fits of the data and from the uncertainties on relative efficiencies estimated with the Monte Carlo simulation. The main uncertainties on the fits of the data derive from the uncertainties of the background models and from the resolution model used in the model of the resonant signals, and from the uncertainty on the contributions of the Cabbibbo suppressed decay modes. The main uncertainties due to the relative efficiencies derive from the uncertainties on the $\Lambda_{c}^{+}, \Lambda_{c}(2595)^{+}$, and $\Lambda_{c}(2625)^{+}$resonant structure, from the average of the relative efficiency for the $\Lambda_{b}^{0} \rightarrow \Lambda_{c}^{+} \rho^{0} \pi^{-}$and $\Lambda_{b}^{0} \rightarrow \Lambda_{c}^{+} \pi^{-} \pi^{+} \pi^{-}$decay modes, which are not separated in this analysis, from the unknown $\Lambda_{b}^{0}$ and $\Lambda_{c}^{+}$polarisations, from the uncertainty on the $\Lambda_{b}^{0}$ production transverse momentum distribution, and from the uncertainty on the $\Lambda_{b}^{0}$ and $\Lambda_{c}^{+}$lifetimes. More details abotu this analysis can be found in [17].

\section{6. $b$ hadron lifetime measurement in the $J / \pi$ decay modes}

CDF has performed a new $b$ hadron lifetime measurement using the decay modes $B^{0} \rightarrow$ $J / \psi K^{* 0}, B^{0} \rightarrow J / \psi K_{s}^{0}, B^{+} \rightarrow J / \psi K^{+}$, and $\Lambda_{b}^{0} \rightarrow J / \psi \Lambda$ reconstructed in $4.3 \mathrm{fb}^{-1}$ of data col- 


\begin{tabular}{l|l}
\hline \hline$\frac{B R\left(\Lambda_{b}^{0} \rightarrow \Lambda_{c}(2595)^{+} \pi^{-} \rightarrow \Lambda_{c}^{+} \pi^{-} \pi^{+} \pi^{-}\right)}{B R\left(\Lambda_{b}^{0} \rightarrow \Lambda_{c}^{+} \pi^{-} \pi^{+} \pi^{-}(\text {all })\right)}$ & $(2.5 \pm 0.6($ stat $) \pm 0.5($ syst $)) \cdot 10^{-2}$ \\
\hline$\frac{B R\left(\Lambda_{b}^{0} \rightarrow \Lambda_{c}(2625)^{+} \pi^{-} \rightarrow \Lambda_{c}^{+} \pi^{-} \pi^{+} \pi^{-}\right)}{B R\left(\Lambda_{b}^{0} \rightarrow \Lambda_{c}^{+} \pi^{-} \pi^{+} \pi^{-}(\text {all })\right)}$ & $\left(6.2 \pm 1.0(\text { stat })_{-0.9}^{+1.0}(\right.$ syst $\left.)\right) \cdot 10^{-2}$ \\
\hline$\frac{B R\left(\Lambda_{b}^{0} \rightarrow \Sigma_{c}(2455)^{++} \pi^{-} \pi^{-} \rightarrow \Lambda_{c}^{+} \pi^{-} \pi^{+} \pi^{-}\right)}{B R\left(\Lambda_{b}^{0} \rightarrow \Lambda_{c}^{+} \pi^{-} \pi^{+} \pi^{-}(\text {all })\right)}$ & $(5.2 \pm 1.1($ stat $) \pm 0.8($ syst $)) \cdot 10^{-2}$ \\
\hline$\frac{B R\left(\Lambda_{b}^{0} \rightarrow \Sigma_{c}(2455)^{0} \pi^{+} \pi^{-} \rightarrow \Lambda_{c}^{+} \pi^{-} \pi^{+} \pi^{-}\right)}{B R\left(\Lambda_{b}^{0} \rightarrow \Lambda_{c}^{+} \pi^{-} \pi^{+} \pi^{-}(\text {all })\right)}$ & $\left(8.9 \pm 2.1(\text { stat })_{-1.0}^{+1.2}(\right.$ syst $\left.)\right) \cdot 10^{-2}$ \\
\hline$\frac{B R\left(\Lambda_{b}^{0} \rightarrow \Lambda_{c}^{+} \rho^{0} \pi^{-}+\Lambda_{c}^{+} 3 \pi(\text { other }) \rightarrow \Lambda_{c}^{+} \pi^{-} \pi^{+} \pi^{-}\right)}{B R\left(\Lambda_{b}^{0} \rightarrow \Lambda_{c}^{+} \pi^{-} \pi^{+} \pi^{-}(\text {all })\right)}$ & $\left(77.3 \pm 3.1(\text { stat })_{-3.3}^{+3.0}(\right.$ syst $\left.)\right) \cdot 10^{-2}$ \\
\hline \hline
\end{tabular}

Table 1: Measured relative Branching Fractions of the resonant $\Lambda_{b}^{0} \rightarrow \Lambda_{c}^{+} \pi^{-} \pi^{+} \pi^{-}$decay modes.
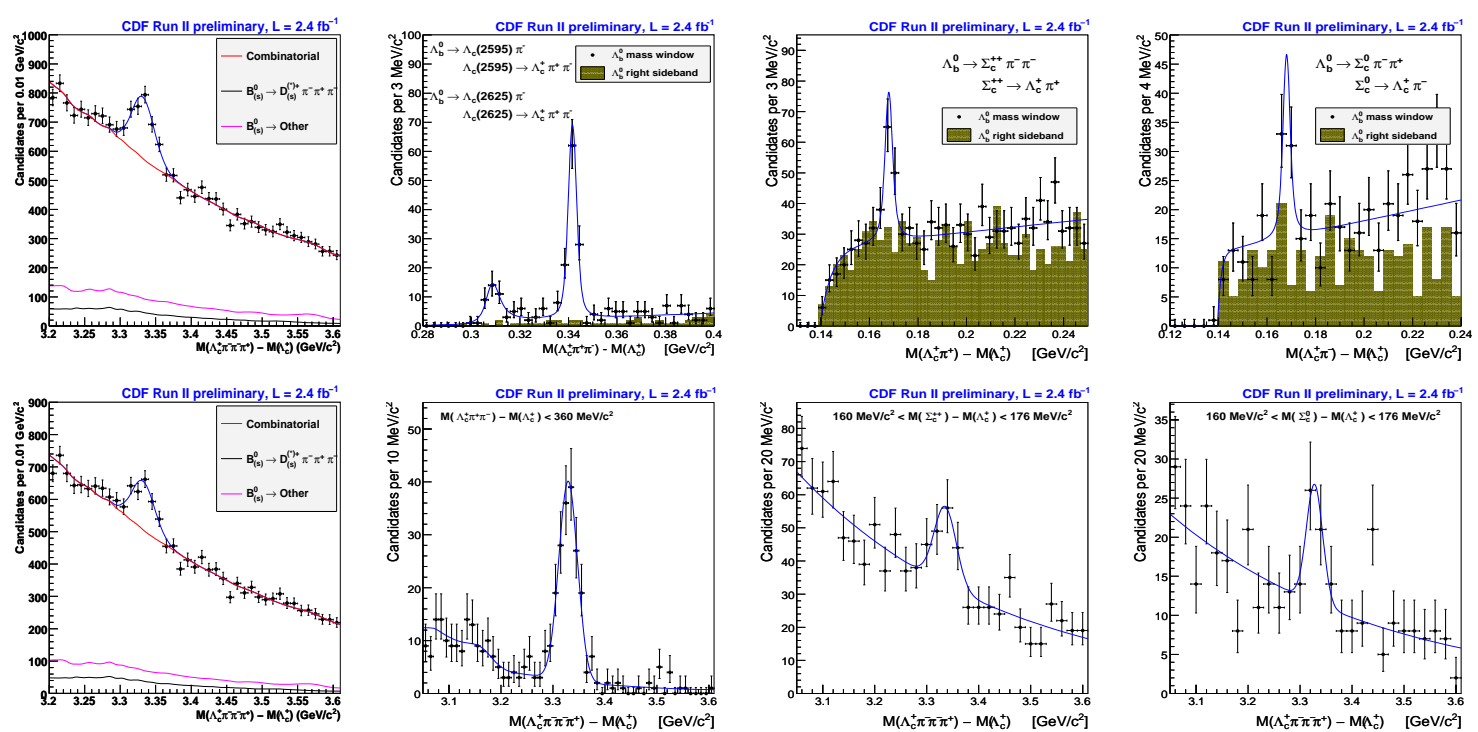

Figure 3: (Top row, first from left) $\Lambda_{b}^{0} \rightarrow \Lambda_{c}^{+} \pi^{-} \pi^{+} \pi^{-}$candidates mass distribution; (Top row, second from left) $\Lambda_{b}^{0} \rightarrow \Lambda_{c}(2595)^{+} \pi^{-}$and $\Lambda_{b}^{0} \rightarrow \Lambda_{c}(2625)^{+} \pi^{-}$candidates in a $\pm 3 \sigma \Lambda_{b}^{0}$ mass window $(\sigma=16$ $\mathrm{MeV} / \mathrm{c}^{2}$ ); (Top row, third from left) $\Lambda_{b}^{0} \rightarrow \Sigma_{c}(2455)^{++} \pi^{-} \pi^{-}$candidates in a $\pm 3 \sigma \Lambda_{b}^{0}$ mass window; (Top row, fourth from left) $\Lambda_{b}^{0} \rightarrow \Sigma_{c}(2455)^{0} \pi^{+} \pi^{-}$candidates in a $\pm 3 \sigma \Lambda_{b}^{0}$ mass window; (Bottom row, first from left) $\Lambda_{b}^{0} \rightarrow \Lambda_{c}^{+} \pi^{-} \pi^{+} \pi^{-}$candidates mass distribution with a veto on the charm baryon resonat decay modes; (Bottom row, second from left) $\Lambda_{b}^{0} \rightarrow \Lambda_{c}^{+} \pi^{-} \pi^{+} \pi^{-}$candidates mass distribution in the $\Lambda_{c}(2595)^{+}$ and $\Lambda_{c}(2625)^{+}$mass window; (Bottom row, third from left) $\Lambda_{b}^{0} \rightarrow \Lambda_{c}^{+} \pi^{-} \pi^{+} \pi^{-}$candidates mass distribution in the $\Sigma_{c}(2455)^{++}$mass window; (Bottom row, fourth from left) $\Lambda_{b}^{0} \rightarrow \Lambda_{c}^{+} \pi^{-} \pi^{+} \pi^{-}$candidates mass distribution in the $\Sigma_{c}(2455)^{0}$ mass window.

lected by the dimuon trigger, which has no biasing effect on the observed proper time distribution. Signal samples of $\approx 45,000 B^{+}, \approx 29,000 B^{0}$, and $\approx 1,700 \Lambda_{b}^{0}$ are reconstructed. The analysis consistes of a maximum likelihood fit that uses information from mass, proper decay time and proper decay time error to extract the lifetime of the hadrons. The use of similar final states and a unified analysis strategy allow partial cancellation of certain systematic uncertainties in the lifetime ratios. The measured lifetimes are the currently most precise determination of the $B^{+}, B^{0}$ and $\Lambda_{b}^{0}$ lifetime. The measured values are $c \tau\left(B^{+}\right)=491.4 \pm 2.6$ (stat.) \pm 2.6 (syst.) $\mu \mathrm{m}$, $c \tau\left(B^{)}\right)=451.7 \pm 3.0$ (stat.) \pm 2.5 (syst.) $\mu \mathrm{m}$, and $c \tau\left(\Lambda_{b}^{0}\right)=460.8 \pm 13.4$ (stat.) \pm 4.1 (syst.) $\mu \mathrm{m}$. [18] 


\section{Conclusions}

In this paper we reviewed the most recent results in the field of B baryons at CDF and D0. These include the observation of the $\Omega_{b}^{-}, \Xi_{b}^{-}, \Sigma_{b}$ and $\Sigma_{b}^{*}$, and the reconstruction of the new $\Lambda_{b}^{0} \rightarrow$ $\Lambda_{c}^{+} \pi^{-} \pi^{+} \pi^{-}$decay mode, and the new measurement of $b$ hadron lifetimes in the $J / \psi$ modes.

\section{References}

[1] D. Acosta et al., the CDF Collaboration, Phys. Rev. D 75, 012010 (2007); T. Aaltonen et al., the CDF Collaboration, Phys. Rev. D 79, 092003 (2009).

[2] T. Aaltonen et al., the CDF Collaboration, Phys. Rev. D 79, 092003 (2009).

[3] A. Sill et al., Nucl. Instrum. Meth. A530:1 (2004).

[4] T. Affolder et al., Nucl. Instrum. Meth. A526:249 (2004).

[5] D. Acosta et al., Nucl. Instrum. Meth. A518:605 (2004).

[6] L. Balka et al., Nucl. Instrum. Meth. A267:272 (1988).

[7] G. Ascoli et al., Nucl. Instrum. Meth. A268:33 (1988).

[8] E. J. Thomson et al., IEEE Trans. on Nucl. Sc. vol. 49, n. 3 (2002).

[9] W. Ashmanskas et al., Nucl. Instrum. Meth. A518: 532, (2004)

[10] V. M. Abazov et al., Instrum. Meth. A565: 463, (2006)

[11] T. Aaltonen et al., Phys. Rev. D80, 072003 (2009).

[12] V. M. Abazov et al., Phys. Rev. Lett. 101, 232002 (2008).

[13] V. M. Abazov et al., Phys. Rev. Lett. 99, 052001 (2007).

[14] T. Aaltonen et al., Phys. Rev. Lett. 9, 202001 (2007).

[15] The CDF B Group Public web page, http://www-cdf.fnal.gov/physics/new/bottom/100721.blessed-Sigmab_6fb/

[16] The CDF B Group Public web page, http://www-cdf.fnal.gov/physics/new/bottom/100701.blessed-Charm_Baryons/

[17] The CDF B Group Public web page, http://www-cdf.fnal.gov/physics/new/bottom/091029.blessed-Lb2Lc3pi-structure/

[18] The CDF B Group Public web page, http://www-cdf.fnal.gov/physics/new/bottom/091217.blessed-JpsiX4.3/ 